\title{
The Thermodynamics of the Ternary System: Water-Potassium Chloride-Calcium Chloride at $25{ }^{\circ} \mathrm{C}$
}

\author{
R. A. Robinson ${ }^{1}$ and A. K. Covington ${ }^{2}$ \\ Institute for Materials Research, National Bureau of Standards, Washington, D.C. 20234
}

(January 26, 1968)

\begin{abstract}
Isopiestic vapor pressure measurements have been made on the system: water-potassium chloridecalcium chloride at $25^{\circ} \mathrm{C}$. The osmotic coefficients of the mixed salt solutions and the activity coefficients of each salt in the presence of the other have been evaluated.
\end{abstract}

Key Words: Activity coefficient, calcium chloride, isopiestic measurements, mixed salt solutions, osmotic coefficients, potassium chloride, vapor pressure.

\section{Introduction}

Isopiestic vapor pressure measurements have been reported for the systems: water-sodium chloridebarium chloride $[1],{ }^{3}$ water-potassium chloride-barium chloride [2], and water-sodium chloride-calcium chloride [3]. Results for the system: water-potassium chloride-calcium chloride are now described.

\section{Definitions}

$M_{B}$ is the molality (moles per kilogram of water) of a solution containing only potassium chloride; it is the "reference" from which isopiestic data are calculated.

$m_{B}, m_{c}$, are the molalities of potassium chloride and calcium chloride, respectively, in a solution containing both these salts and in isopiestic (vapor phase) equilibrium with the potassium chloride solution of molality $M_{B}$.

$m=m_{B}+1.5 m_{C}$.

$R=M_{B} / m$ is the isopiestic ratio.

$x_{c}=1.5 \mathrm{~m} / \mathrm{m}$.

$I=m_{B}+3 m_{C}$ is the total ionic strength of the solution.

$y_{B}=m_{B} / I$.

$y_{C}=3 m_{c} / I$.

$\gamma_{B}, \gamma_{C}$, are the activity coefficients of potassium chloride and calcium chloride, respectively, in the mixed salt solution.

'Present address: Department of Chemistry, State University of New York at Binghamton. N.Y. 13901

hamton. N.Y. 13901 . 1966 ) on leave from the University of Newcastle upon Tyne, England.

${ }^{3}$ Figures in brackets indicate the literature references at the end of this paper.
$\gamma_{B}^{\circ}$ is the activity coefficient of potassium chloride in a solution containing potassium chloride only at the same total ionic strength $I=m_{B}+3 m_{C}$ as the mixed solution.

$\gamma_{c}^{\circ}$ is the activity coefficient of calcium chloride in a solution containing only calcium chloride at the same total ionic strength, $I$.

$\varphi=-55.51 \ln a_{w} /\left(2 m_{B}+3 m_{C}\right)=-55.51 \ln$ $a_{w} /\left[\left(y_{B}+1\right) I\right]$ is the osmotic coefficient of the mixed salt solution.

$\varphi_{B}^{\circ}$ is the osmotic coefficient of the solution containing potassium chloride only at the same total ionic strength as that of the mixed salt solution.

$\varphi_{C}^{\circ}$ is the osmotic coefficient of the solution containing calcium chloride only at the same total ionic strength as the mixed salt solution.

\section{Experimental Procedure and Results}

The salts were portions of those used in earlier work $[2,3]$. Isopiestic vapor pressure measurements were made in the manner described previously [1]. The results are given in table 1.

\section{Discussion}

The immediate result of an isopiestic measurement is the information that a solution containing two salts of known molality has the same aqueous vapor pressure as a solution of one of the salts, again at a known molality. Such information is readily transformed into osmotic coefficient data. Activity coefficient data, 
TABLE 1. Isopiestic data for the system: potassium chloride (B) - calcium chloride (C)

\begin{tabular}{|c|c|c|c|c|c|c|c|c|c|c|c|}
\hline Set & $M_{B}$ & $m_{B}$ & $m_{C}$ & $\varphi$ (obs.) & $\varphi($ calc. $)$ & Set & $M_{B}$ & $m_{B}$ & $m_{C}$ & $\varphi$ (obs.) & $\varphi$ (calc.) \\
\hline \multirow[t]{8}{*}{1} & 0.7560 & 0.5219 & 0.1564 & 0.8964 & 0.8942 & & & 0.6726 & 1.2065 & 1.1193 & 1.1182 \\
\hline & & .2903 & .3087 & .9002 & .8990 & & & & 1.5264 & 1.2136 & 1.2109 \\
\hline & & .1347 & .4072 & .9095 & .9055 & & 2.9694 & 1.8591 & 0.6052 & 1.0044 & 1.0048 \\
\hline & & & .4948 & .9141 & .9140 & & & 1.0832 & 1.0040 & 1.0733 & 1.0728 \\
\hline & 0.7577 & 0.6384 & 0.0789 & 0.8987 & 0.8944 & & & 0.3690 & 1.3528 & 1.1588 & 1.1570 \\
\hline & & .4168 & .2267 & .8980 & .8957 & & & & & & \\
\hline & & .1929 & .3723 & .9047 & .9031 & 7 & 3.4262 & 2.8230 & 0.3250 & 0.9815 & 0.9811 \\
\hline & & & & & & & & 1.7945 & .8579 & 1.0545 & 1.0552 \\
\hline \multirow[t]{5}{*}{2} & 1.1535 & 0.9288 & 0.1440 & 0.9051 & 0.9041 & & & 0.8574 & 1.3132 & 1.1494 & 1.1510 \\
\hline & & .5890 & .3579 & .9204 & .9207 & & 3.4611 & 2.2892 & 0.6221 & 1.0196 & 1.0193 \\
\hline & & .2704 & .5525 & .9427 & .9434 & & & 1.2082 & 1.1605 & 1.1142 & 1.1151 \\
\hline & & & .7140 & .9675 & .9691 & & & 0.3930 & 1.5399 & 1.2156 & 1.2153 \\
\hline & & & & & & & & & 1.7164 & 1.2761 & 1.2742 \\
\hline \multirow[t]{7}{*}{3} & 1.5135 & 1.3037 & 0.1310 & 0.9108 & 0.9117 & & & & & & \\
\hline & & 0.9350 & .3559 & .9302 & .9327 & 8 & 3.7176 & 2.7254 & 0.5244 & 1.0125 & 1.0128 \\
\hline & & .5999 & .5518 & .9571 & .9569 & & & 1.6537 & 1.0560 & 1.0983 & 1.0982 \\
\hline & & & .8952 & 1.0175 & 1.0180 & & & $* 0.5957$ & 1.5476 & 1.2190 & \\
\hline & 1.5240 & 1.0898 & 0.2681 & 0.9223 & 0.9240 & & 3.7221 & 3.1867 & 0.2866 & 0.9845 & 0.9848 \\
\hline & & 0.7744 & .4569 & .9427 & .9448 & & & 2.2269 & .7784 & 1.0489 & 1.0492 \\
\hline & & .4029 & .6734 & .9740 & .9759 & & & 1.1373 & 1.3036 & 1.1513 & 1.1529 \\
\hline \multirow{7}{*}{4} & 20809 & 2658 & 04755 & 0.9613 & 0.9641 & & & & 1.8128 & 1.3094 & 1.3089 \\
\hline & & $\begin{array}{l}1.2050 \\
0.7063\end{array}$ & $\begin{array}{r}0.4855 \\
.7865\end{array}$ & 1.0087 & $\begin{array}{l}0.0041 \\
1.0118\end{array}$ & 9 & 4.3263 & 3.7758 & 0.2844 & 1.0031 & 1.0018 \\
\hline & & .2483 & 1.0229 & 1.0672 & 1.0600 & & & *2.6197 & .8587 & 1.0784 & \\
\hline & 2.1530 & 1.7491 & 0.2373 & 0.9370 & 0.9383 & & & ${ }^{*} 1.6439$ & 1.3151 & 1.1653 & \\
\hline & & 1.0520 & .6320 & .9862 & .9881 & & & & 2.0262 & 1.3871 & 1.3859 \\
\hline & & 0.4806 & .9403 & 1.0431 & 1.0434 & & 4.3543 & 3.2196 & 0.5776 & 1.0394 & 1.0380 \\
\hline & & & 1.1896 & 1.1050 & 1.1041 & & & *1.9778 & 1.1736 & 1.1360 & W. \\
\hline \multirow[t]{7}{*}{5} & 2.7872 & 1.9415 & 0.4654 & 0.9833 & 0.9813 & & & *0.5997 & 1.7831 & 1.2970 & \\
\hline & & 0.6796 & 1.1226 & 1.0982 & 1.0954 & 10 & 4.6033 & 3.8770 & 0.3749 & 1.0188 & 1.0217 \\
\hline & & .3588 & 1.2791 & 1.1397 & 1.1349 & & & *2.4736 & 1.0524 & 1.1161 & \\
\hline & 2.7986 & 2.3180 & 0.2674 & 0.9589 & 0.9585 & & & *1.0844 & 1.6724 & 1.2607 & \\
\hline & & 1.4805 & .7194 & 1.0185 & 1.0178 & & & & 2.1195 & 1.4226 & 1.4206 \\
\hline & & 1.0515 & .9406 & 1.0586 & 1.0567 & & 4.6201 & ${ }^{*} 3.1511$ & 0.7405 & 1.0656 & \\
\hline & & & & & & & & ${ }^{*} 1.5840$ & 1.4625 & 1.2022 & \\
\hline \multirow[t]{2}{*}{6} & 2.9689 & 2.4640 & 0.2806 & 0.9632 & 0.9645 & & & $* 0.5200$ & 1.9134 & 1.3396 & \\
\hline & & 1.5330 & .7747 & 1.0310 & 1.0307 & & & & & & \\
\hline
\end{tabular}

*Not used in computing values of $\beta_{0}$ in eq (26).

however, have to be obtained indirectly. One method achieves this objective by means of the McKayPerring procedure [4]. It was shown earlier [1] that if the isopiestic ratios, $R$, can be expressed in terms of the ionic fraction, $x_{c}$, by means of the equation

$$
R=1+a x_{C},
$$

the application of the McKay-Perring method is very simple. Even if an additional term is needed,

$$
R=1+a x_{C}+b x^{2},
$$

it is still possible to use the method.

Figure 1 shows some of the isopiestic data obtained in the present work. It will be observed that there is considerable curvature in the plots, particularly for those corresponding to solutions of low total ionic strength. It was found that eq (2) was inadequate to represent the experimental results and even the addition of a further term in $x_{C}^{3}$ gave only slightly better agreement. However, even if the equation

$$
R=1+a x_{C}+b x_{C}^{2}+c x_{C}^{3}
$$

was adequate, it would be difficult to handle the integration needed in the McKay-Perring method.

An alternative, but equivalent, approach, based on the work of Scatchard [5], is now presented. As this should have wide application to the study of mixed salt solutions, it will be described in some detail.

Consider, first, a solution of two salts each of the 1:1 charge type. Let the activity coefficients of the salts in solutions of constant total ionic strength ( $I=m_{B}+m_{C}$ if the salts are of the $1: 1$ charge type) be capable of representation by the equations,

$$
\begin{aligned}
& \ln \gamma_{B}=\ln \gamma_{B}^{\circ}+Q_{B} y_{C}+R_{B} y_{C}^{2} \\
& \ln \gamma_{C}=\ln \gamma_{C}^{\circ}+Q_{C} y_{B}+R_{C} y_{B}^{2} .
\end{aligned}
$$

Here $y_{B}=m_{B} / I, y_{C}=m_{C} / I$ for salts of this charge type. $Q_{B}, Q_{C}, R_{B}$, and $R_{C}$ may be functions of $I$ but not of $y_{B}, y_{C}$ individually.

It has been shown, by the use of the Gibbs-Duhem equation, [2, 6, 7] that, if eqs (4) and (5) hold, the osmotic coefficient of the solution is given as 


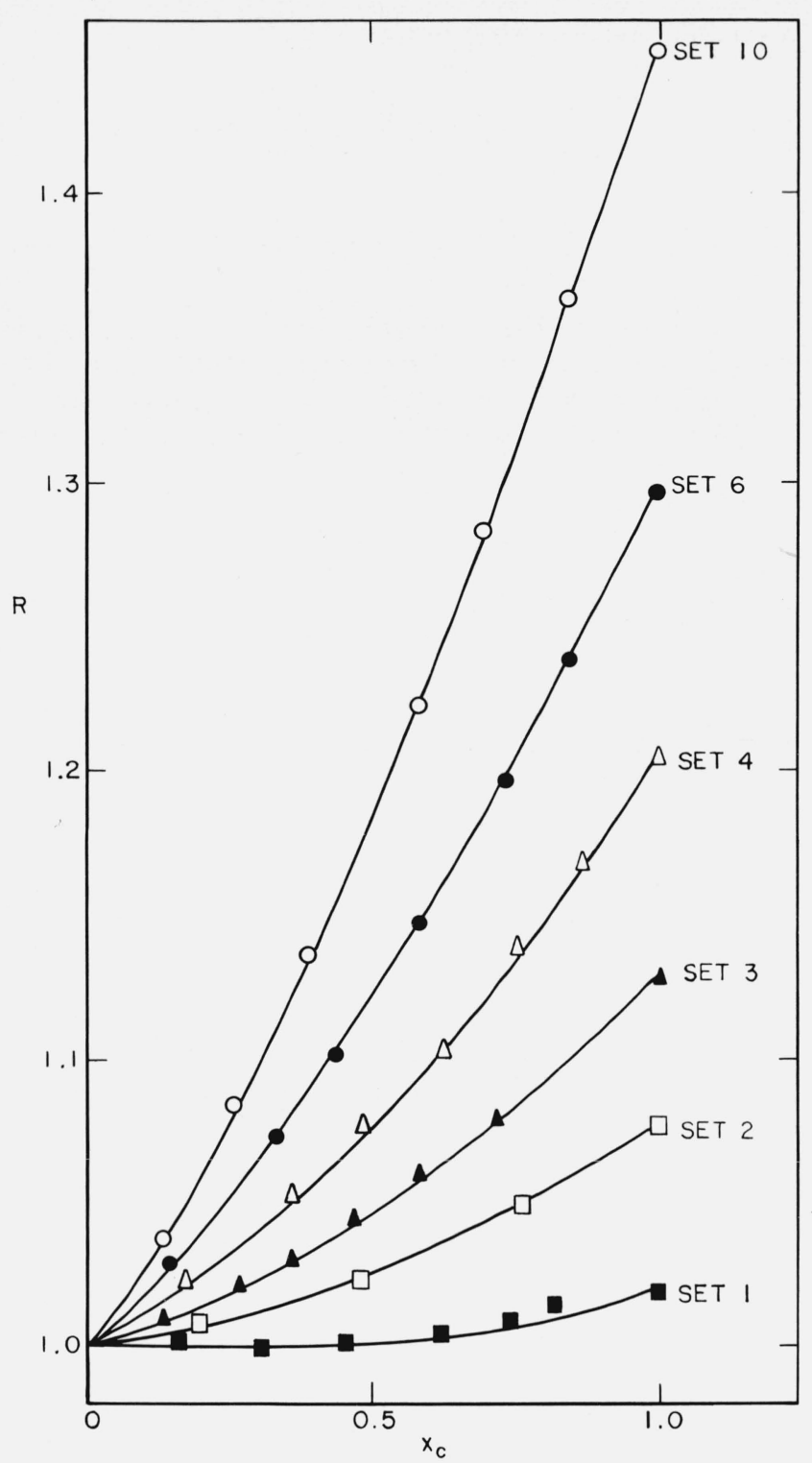

FIGURE 1. Isopiestic ratio $(\mathrm{R})$ versus ionic fraction $\left(\mathrm{x}_{\mathrm{C}}\right)$ of calcium chloride.

$\varphi=\varphi_{B}^{\circ}+y_{C} Q_{B}-\frac{1}{2} y_{C}^{2}\left(Q_{B}+Q_{C}\right)$

$$
+y_{C}^{2}\left(1-\frac{2}{3} y_{C}\right)\left(R_{B}-R_{C}\right)
$$

and, by symmetry,

$\varphi=\varphi_{C}^{\circ}+y_{B} Q_{C}-\frac{1}{2} y_{B}^{2}\left(Q_{B}+Q_{C}\right)$

$$
+y_{B}^{2}\left(1-\frac{2}{3} y_{B}\right)\left(R_{C}-R_{B}\right)
$$

Combining eqs (6) and (7), we get

$$
\begin{aligned}
\varphi=y_{B} \varphi_{B}^{0}+y_{C} \varphi_{C}^{0}+ & \frac{1}{2} y_{B} y_{C}\left(Q_{B}+Q_{C}\right) \\
& +\frac{1}{3} y_{B} y_{C}\left(y_{B}-y_{C}\right)\left(R_{C}-R_{B}\right) .
\end{aligned}
$$

Since $\varphi_{B}^{0}$ and $\varphi_{C}^{0}$ depend only upon properties of solutions containing a single salt, the departure from ideality on mixing the single salt solutions can be represented as

$$
\begin{aligned}
\Delta & =2 \varphi-2 y_{B} \varphi_{B}^{0}-2 y_{C} \varphi_{C}^{0} \\
& =y_{B} y_{C}\left(Q_{B}+Q_{C}\right)+\frac{2}{3} y_{B} y_{C}\left(y_{B}-y_{C}\right)\left(R_{C}-R_{B}\right) .
\end{aligned}
$$

If $R_{B}=R_{C}=0$ and $\varphi$ can be described entirely in terms of $Q_{B}$ and $Q_{C}$ (the case of the Harned Rule) a plot of $\Delta$ against $y_{C}$ should be symmetrical about the $y_{B}=y_{C}=0.5$ axis. The $\left(R_{C}-R_{B}\right)$ term allows for a possible skew in the plot.

Figure 2 shows some data for the system: watersodium chloride $(B)$-potassium chloride $(C)[8]$. The value of $\Delta$ was calculated using the data in series 6 of this reference. It is true that the values of $\Delta$ are taken from data where the total ionic strength varied from 4.0 to 4.5 and, therefore, do not satisfy exactly the condition of constant total ionic strength. However, the figure suffices to show that $\Delta$ should be capable of expression by a term in $y_{B} y_{C}$ with only a small term in $y_{B} y_{C}\left(y_{B}-y_{C}\right)$.

In Scatchard's equations for a solution containing two $1: 1$ salts, the osmotic coefficient of the solution is given by the equation

$$
\begin{aligned}
2 \varphi=2 y_{B} \varphi_{B}^{0}+2 y_{C} \varphi_{C}^{0} & +y_{B} y_{C} \beta_{0} \\
& +y_{B} y_{C}\left(y_{B}-y_{C}\right) \beta_{1}+\ldots .
\end{aligned}
$$

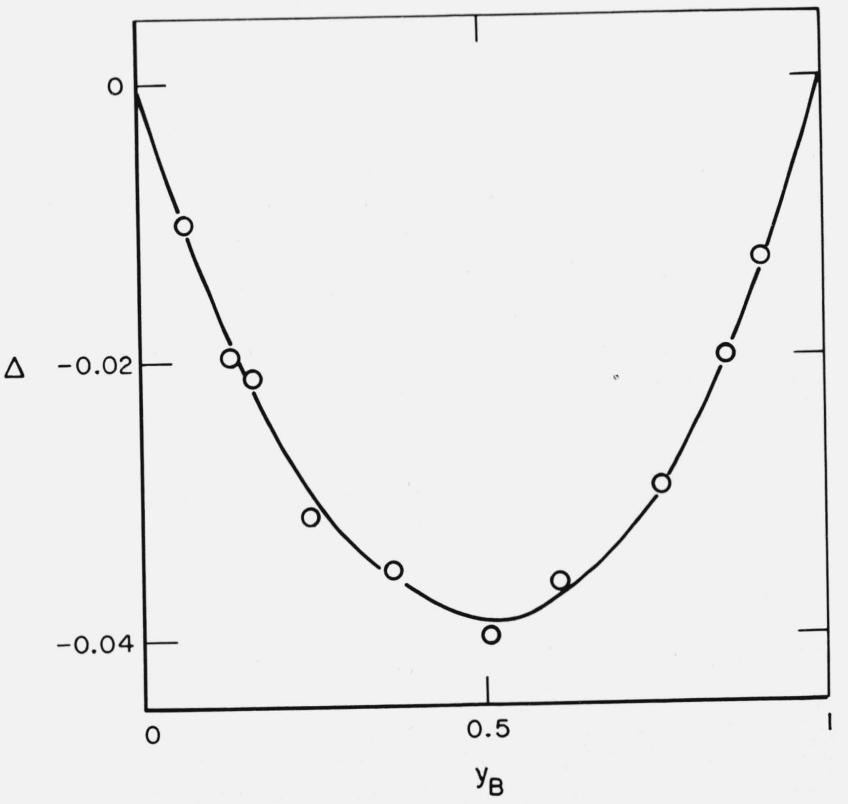

FigURE 2. The function $\Delta=2 \varphi-2 \mathrm{y}_{\mathrm{B}} \varphi_{\mathrm{B}}^{0}-2 \mathrm{y}_{\mathrm{C}} \varphi_{\mathrm{C}}^{0}$ for the system: water-sodium chloride $(\mathrm{B})$-potassium chloride (C) at $25^{\circ} \mathrm{C}$. 
with

$$
\beta_{0}=b_{01} m+b_{02} m^{2}+\ldots
$$

and

$$
\beta_{1}=b_{12} m^{2}+\ldots
$$

Unlike the $Q$ and $R$ terms of eqs (4) and (5), $b_{01}, b_{02}$, and $b_{12}$ are independent of $m$.

The activity coefficient of salt $B$ is given by the equation

$2 \ln \gamma_{B} / \gamma_{B}^{0}=\left[2\left(\varphi_{C}^{0}-\varphi_{B}^{0}\right)+\beta_{0}+\beta_{1}\right] y_{C}$

$$
+\left[\left(B_{0}-\beta_{0}\right)+3\left(B_{1}-\beta_{1}\right)\right] y_{C}^{2}-2\left(2 B_{1}-\beta_{1}\right) y_{C}^{3}
$$

and that of salt $C$ by the equation

$$
\begin{aligned}
& 2 \ln \gamma_{C} / \gamma_{C}^{0}=\left[2\left(\varphi_{B}^{0}-\varphi_{C}^{0}\right)+\beta_{0}-\beta_{1}\right] y_{B} \\
& +\left[\left(B_{0}-\beta_{0}\right)-3\left(B_{1}-\beta_{1}\right)\right] y_{B}^{2}+2\left(2 B_{1}-\beta_{1}\right) y_{B}^{3} .
\end{aligned}
$$

Here, $B_{0}=m d \beta_{0} / d m$ and $B_{1}=m d \beta_{1} / d m$. If $\beta_{1}$ does not require terms higher than $m^{2}$, then the coefficients of the $y_{B}^{3}$ and $y_{C}^{3}$ terms are zero.

Equation (10) can be written

$$
\begin{aligned}
\Delta=2 \varphi-2 y_{B} \varphi_{B}^{0}-2 y_{C} \varphi_{C}^{0}= & y_{B} y_{C}\left(b_{01} m+b_{02} m^{2}\right) \\
& +y_{B} y_{C}\left(y_{B}-y_{C}\right) b_{12} m^{2} .
\end{aligned}
$$

The procedure then is to use isopiestic data to calculate the osmotic coefficients of mixed salt solutions at all available values of $\mathrm{y}_{B}$ and $m$. The coefficients $b_{01}, b_{02}$, and $b_{12}$ are then evaluated by the method of least squares. These, together with the corresponding values of $B_{0}$ and $B_{1}$, are then substituted in eqs (13) and (14) to get values of the activity coefficients. The application of these equations to the system: water-sodium chloride-potassium chloride has already been considered [9].

For the potassium chloride $(B)$-calcium chloride $(C)$ system, it is convenient to write

$$
\begin{gathered}
2 \ln \gamma_{B}=2 \ln \gamma_{B}^{0}+Q_{B} y_{C}+R_{B} y_{C}^{2} \\
\ln \gamma_{C}=\ln \gamma_{C}^{0}+Q_{C} y_{B}+R_{C} y_{B}^{2} .
\end{gathered}
$$

The equation for the osmotic coefficient now becomes

$$
\begin{array}{r}
\left(y_{B}+1\right) \varphi=2 y_{B} \varphi_{B}^{0}+y_{C} \varphi_{C}^{0}+\frac{1}{2} y_{B} y_{C}\left(Q_{B}+Q_{C}\right) \\
+\frac{1}{3} y_{B} y_{C}\left(y_{B}-y_{C}\right)\left(R_{C}-R_{B}\right) .
\end{array}
$$

A convenient deviation function

$$
\Delta=\left(y_{B}+1\right) \varphi-2 y_{B} \varphi_{B}^{0}-y_{C} \varphi_{C}^{0}=\frac{1}{2} y_{B} y_{C}\left(Q_{B}+Q_{C}\right)
$$

$$
+\frac{1}{3} y_{B} y_{C}\left(y_{B}-y_{C}\right)\left(R_{C}-R_{B}\right)
$$

now represents the departure from ideality when solutions of potassium chloride and calcium chloride are mixed at constant total ionic strength.

Table 2 gives some values of $\Delta$ for this system, utilizing the data of set 6 of table 1 . The values of $\Delta$ are plotted in figure 3 . It will be observed that $\Delta$ is smaller for this system than for sodium chloride-potassium chloride mixtures (see fig. 2). The total ionic strength of these solutions lay between 3.3 and 4.4 ; therefore, they be no means satisfy the condition of constant total ionic strength. The only purpose of figure 3 is to suggest that departures from ideality for solutions of this salt pair are comparatively small.

Scatchard's equations for a system of this charge type become

$$
\left(y_{B}+1\right) \varphi=2 y_{B} \varphi_{B}^{0}+y_{C} \varphi_{C}^{0}+y_{B} y_{C} \beta_{0}+y_{B} y_{C}\left(y_{B}-y_{C}\right) \beta_{1}
$$

$2 \ln \gamma_{B} / \gamma_{B}^{0}=\left[\left(\varphi_{C}^{0}-1\right)-2\left(\varphi_{B}^{0}-1\right)+\beta_{0}+\beta_{1}\right] y_{C}$

$$
+\left[\left(B_{0}-\beta_{0}\right)+3\left(B_{1}-\beta_{1}\right)\right] y_{c}^{2}-2\left(2 B_{1}-\beta_{1}\right) y_{c}^{3}
$$

TABLE 2. Osmotic coefficients of solutions of potassium chloride (B) + calcium chloride $(\mathrm{C})$ at $25^{\circ} \mathrm{C}$

\begin{tabular}{c|r|r|c|c|c}
\hline \hline$m_{B}$ & $m_{C}$ & $y_{B}$ & $\varphi$ & $\Delta^{\mathrm{a}}$ & $\Delta_{\text {(calc) }}{ }^{\mathrm{b}}$ \\
\hline & & & & & \\
\hline 1.4640 & 0.2806 & 0.7454 & 0.9632 & -0.0030 & -0.0029 \\
1.5591 & .6052 & .5059 & 1.0044 & -.0047 & -.0056 \\
1.0832 & .7747 & .3974 & 1.0310 & -.0059 & -.0061 \\
0.6726 & 1.0040 & .2645 & 1.0733 & -.0065 & -.0058 \\
0.3690 & 1.2065 & .1567 & 1.1193 & -.0051 & -.0043 \\
& 1.3528 & .0833 & 1.1588 & -.0022 & -.0027 \\
\hline
\end{tabular}

${ }^{\text {a }} \Delta=\left(y_{B}+1\right) \varphi-2 y_{B} \varphi_{B}^{0}-y_{C} \varphi_{C}^{0}$

${ }^{\mathrm{b}} \Delta_{\text {(calc) }}=-0.0225 y_{B} y_{C}+0.0150 y_{C}\left(y_{B}-y_{C}\right)$

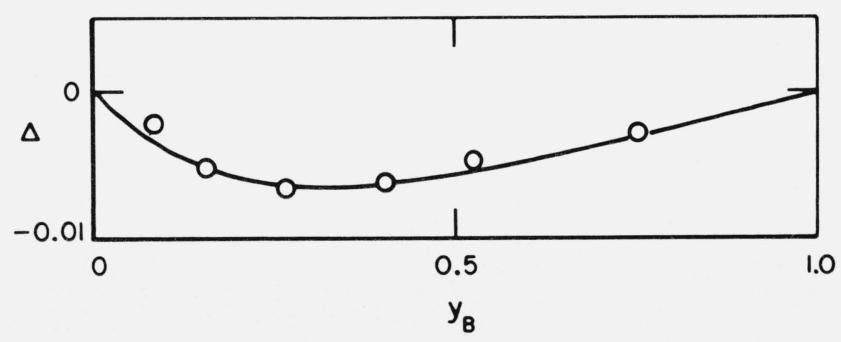

FigURE 3. The function $\Delta=\left(\mathrm{y}_{\mathrm{B}}+1\right) \varphi-2 \mathrm{y}_{\mathrm{B}} \varphi_{\mathrm{B}}^{0}-\mathrm{y}_{\mathrm{C}} \varphi_{\mathrm{C}}^{0}$ for the system: water-potassium chloride (B)-calcium chloride (C) at $25^{\circ} \mathrm{C}$. 
$\ln \gamma_{C} / \gamma_{C}^{0}=\left[2\left(\varphi_{B}^{0}-1\right)-\left(\varphi_{C}^{0}-1\right)+\beta_{0}-\beta_{1}\right] y_{B}$

$+\left[\left(B_{0}-\beta_{0}\right)-3\left(B_{1}-\beta_{1}\right)\right] y_{B}^{2}+2\left(2 B_{1}-\beta_{1}\right) y_{B}^{3}$.

The osmotic coefficients of solutions of potassium chloride alone [10] were expressed as a polynomial in $I$ by means of the equation

$$
\begin{aligned}
2\left(\varphi_{B}^{0}-1\right)=\varphi^{D H}-2 & \left(0.03204 I-0.02622 I^{2}\right. \\
+ & \left.0.00562 I^{3}-0.000459 I^{4}\right)
\end{aligned}
$$

and those for calcium chloride alone $[3,11]$ by means of the equation

$$
\left(\varphi_{C}^{0}-1\right)=\varphi^{D H}+0.05840 I+0.00553 I^{2}-0.000068 I^{3} .
$$

Here, $\varphi^{D H}$ is the Debye-Hückel contribution to the osmotic coefficient and, for aqueous solutions at $25{ }^{\circ} \mathrm{C}$, can be written

$$
\varphi^{D H}=-\frac{2.34164}{\rho^{3} I}\left[(1+x)-2 \ln (1+x)-(1+x)^{-1}\right]
$$

where $x=\rho I^{1 / 2}$ and $\rho=1.5$ mole $^{-1 / 2} \mathrm{~kg}^{1 / 2}$.

With the values of $\varphi_{B}^{0}$ and $\varphi_{C}^{0}$ calculated with the aid of eqs (23) and (24), and the osmotic coefficient data for the mixed salt solutions given in table 1 , it was found, by the method of least squares, that a fair approximation to the experimental values could be obtained by putting $\beta_{0}=b_{01} I+b_{02} I^{2}=0.03330 I-0.01036 I^{2}$. The fit is perhaps not as good as one might wish, the standard deviation being 0.0021 in $\varphi$. The introduction of a further term, however, $\beta_{0}=0.03340 \mathrm{I}$ $-0.01045 I^{2}, \beta_{1}=-0.00035 I^{2}$, did not improve the agreement. It was decided, therefore, that the simpler equation should be adopted. The equations now become

$$
\left(y_{B}+1\right) \varphi=2 y_{B} \varphi_{B}^{0}+\varphi_{C}^{0}+y_{B} y_{C} \beta_{0}
$$

$2 \ln \gamma_{B} / \gamma_{B}^{0}=\left[\left(\varphi_{C}^{0}-1\right)-2\left(\varphi_{B}^{0}-1\right)+\beta_{0}\right] y_{C}$

$$
+\left(B_{0}-\beta_{0}\right) y_{C}^{2}
$$

$\ln \gamma_{C} / \gamma_{C}^{0}=\left[2\left(\varphi_{B}^{0}-1\right)-\left(\varphi_{C}^{0}-1\right)+\beta_{0}\right] y_{B}$

with

$$
+\left(B_{0}-\beta_{0}\right) y_{B}^{2}
$$

$$
\beta_{0}=0.03330 I-0.01036 I^{2}, B_{0}=0.03330 I-0.00518 I^{2},
$$

and

$$
\left(B_{0}-\beta_{0}\right)=0.00518 I^{2} \text {. }
$$

\begin{tabular}{|c|c|c|c|}
\hline$y_{C}$ & $\varphi$ & $\log \gamma_{B} / \gamma_{B}^{0}$ & $\log \gamma_{C} / \gamma_{C}^{0}$ \\
\hline$I=1$ & & & \\
\hline 0 & 0.8962 & 0 & -0.0251 \\
\hline 0.2 & .8963 & 0.0048 & -.0204 \\
\hline .4 & .8952 & .0096 & -.0156 \\
\hline .6 & .8925 & .0146 & -.0106 \\
\hline .8 & .8874 & .0196 & -.0054 \\
\hline 1.0 & .8784 & .0248 & 0 \\
\hline$I=2$ & & & \\
\hline 0 & 0.9122 & 0 & -0.0375 \\
\hline 0.2 & .9194 & 0.0070 & -.0314 \\
\hline .4 & .9271 & .0144 & -.0246 \\
\hline .6 & .9356 & .0221 & -.0171 \\
\hline .8 & .9452 & .0302 & -.0089 \\
\hline 1.0 & .9566 & .0387 & 0 \\
\hline$I=3$ & & & \\
\hline 0 & 0.9375 & 0 & -0.0519 \\
\hline 0.2 & .9503 & 0.0082 & -.0447 \\
\hline .4 & .9661 & .0172 & -.0360 \\
\hline .6 & .9859 & .0270 & -.0256 \\
\hline .8 & 1.0119 & .0377 & -.0136 \\
\hline 1.0 & 1.0478 & .0491 & 0 \\
\hline$I=4$ & & & \\
\hline 0 & 0.9644 & 0 & -0.0735 \\
\hline 0.2 & .9820 & 0.0089 & -.0646 \\
\hline .4 & 1.0056 & .0192 & -.0527 \\
\hline .6 & 1.0378 & .0309 & -.0380 \\
\hline .8 & 1.0828 & .0441 & -.0205 \\
\hline 1.0 & 1.1485 & .0587 & 0 \\
\hline$I=5$ & & & \\
\hline 0 & 0.9977 & 0 & -0.0980 \\
\hline 0.2 & 1.0184 & 0.0085 & -.0874 \\
\hline .4 & 1.0489 & .0193 & -.0723 \\
\hline 6 & 1.0935 & .0323 & -.0527 \\
\hline .8 & 1.1590 & .0476 & -.0286 \\
\hline 1.0 & 1.2582 & .0652 & 0 \\
\hline
\end{tabular}

Values of the osmotic coefficients calculated by means of eq (26) are given in the last column of table 1. Some data in table 1 are marked with an asterisk; in these cases the total ionic strength was greater than that of saturated potassium chloride solution and it is doubtful if eq (23) is applicable; these data
TABLE 3. Osmotic and activity coefficients of the system water potassium chloride-calcium chloride at $25^{\circ} \mathrm{C}$

were not used in calculating values of $b_{01}$ and $b_{02}$ by the method of least squares.

Activity coefficients for the mixed salt solutions have been calculated using eqs (27) and (28); values of $\log \gamma_{B} / \gamma_{B}^{0}$ and $\log \gamma_{C} / \gamma_{C}^{0}$ are given in table 3 and plotted in figures 4 and 5 . As will be seen from these figures, plots of $\log \gamma_{B}$ against $y_{C}$ and of $\log \gamma_{C}$ against $y_{B}$ are by no means linear except at the lowest value of the total ionic strength, $I=1$, where the curvature is slight. Moreover, the variation of the activity coefficient is large; thus at $I=4, \log \gamma_{B}^{0}=-0.2368$ for potassium chloride in its own solution, whereas $\log \gamma_{B}^{\text {tr }}=-0.1781$ for the "trace" activity coefficient of potassium chloride in the limit when the solution contains only calcium chloride, a difference of 0.0587 . The corresponding quantities for calcium chloride are $\log \gamma_{C}^{0}=-0.2502$ and $\log \gamma_{C}^{\mathrm{tr}}=-0.3237$, a difference of 0.0735 .

These differences are much larger than those found for other systems of this charge type, as is emphasized by figure 6 . It will be seen that the next largest variation is found with barium chloride in potassium chloride solution, where $\log \gamma_{C}^{0} / \gamma_{C}^{\mathrm{tr}}=0.021$. Moreover, 


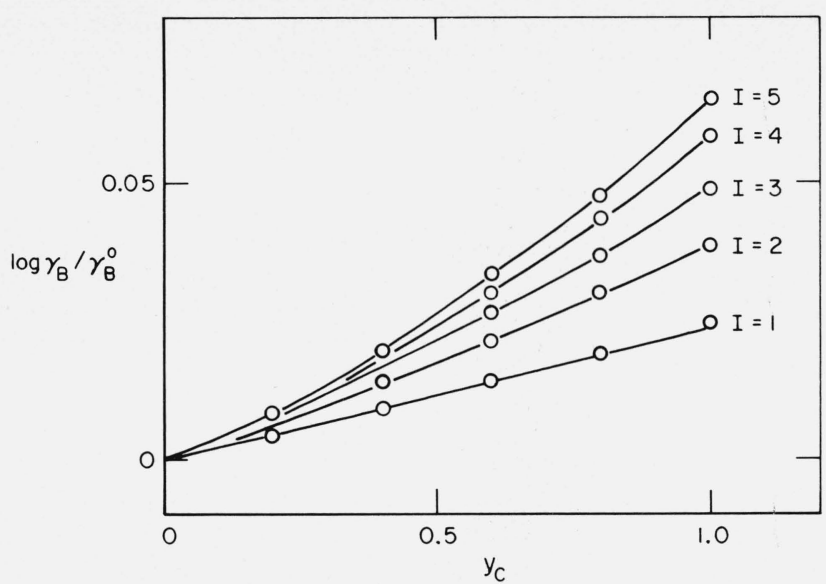

FIGURE 4. Variation of the activity coefficient of potassium chloride in potassium chloride-calcium chloride mixtures at constant total ionic strength.

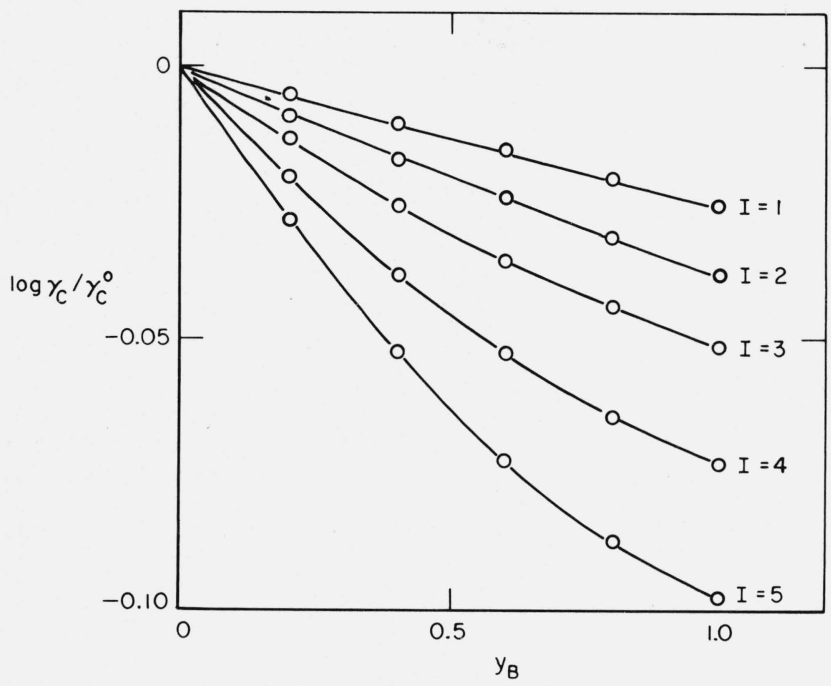

FIGURE 5. Variation of the activity coefficient of calcium chloride in potassium chloride-calcium chloride mixtures at constant total ionic strength.

there is a marked curvature in the two plots for the potassium chloride-calcium chloride system whereaś, for the other three systems, any curvature in the plots must have been small.

Kirgintsev and Luk'yanov [12] have reported isopiestic vapor pressure measurements of this system. They had nine mixed salt solutions of different potas. sium chloride to calcium chloride ratios and these were equilibrated with six reference potassium chloride solutions of molalities ranging from 1.857 to $4.217 \mathrm{~m}$.

Their results are in good agreement with ours. This can be illustrated by means of figure 7 . We have used our data from set 7 of table 1 for six solutions in equilibrium with either 3.4262 or $3.4611 \mathrm{~m}$ potassium chloride and also the data of Kirgintsev and Luk'yanov for nine solutions in equilibrium with $3.362 \mathrm{~m}$ potassium chloride and calculated the deviation function, $\Delta$,
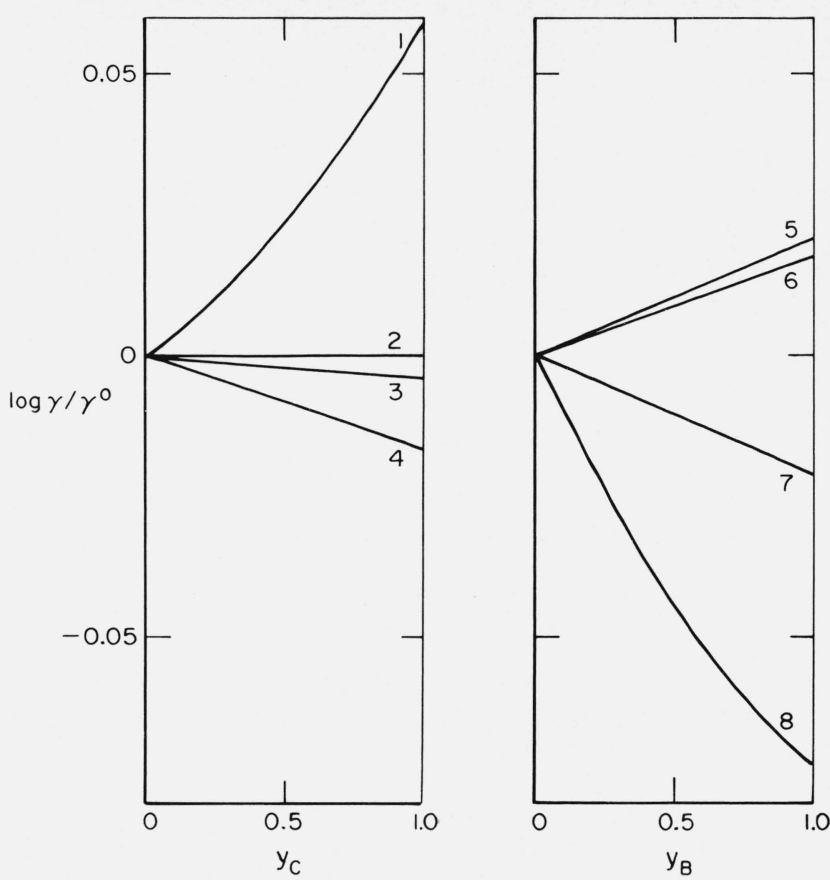

FIGURE 6. Activity coefficients in solutions at a constant total ionic strength of $\mathrm{I}=4$.

1. Potassium chloride in calcium chloride solution.

2. Sodium chloride in calcium chloride solution.

3. Potassium chloride in barium chloride solution.

3. Potassium chloride in barium chloride solution.
4. Sodium chloride in barium chloride solution.

4. Sodium chloride in barium chloride solution.

5. Barium chloride in sodium chloride solution.

6. Calcium chloride in sodium chloride solution.

7. Barium chloride in potassium chloride solution.

8. Calcium chloride in potassium chloride solution.

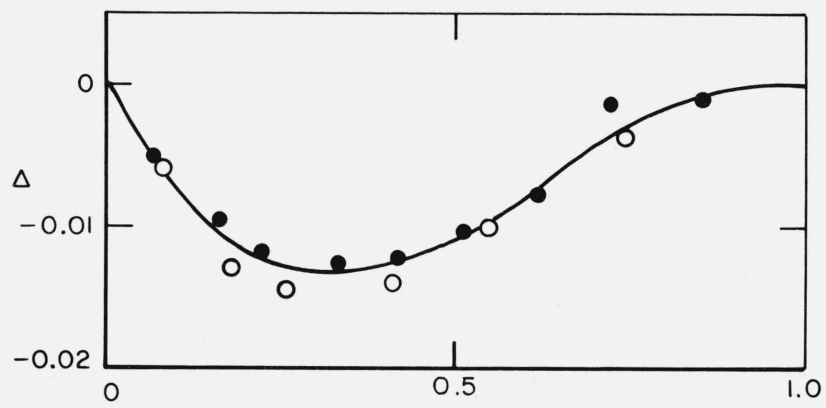

Figure 7. The function $\Delta=\left(\mathrm{y}_{\mathrm{B}}+1\right) \varphi-2 \mathrm{y}_{\mathrm{B}} \varphi_{\mathrm{B}}^{0}-\mathrm{y}_{\mathrm{C}} \varphi_{\mathrm{C}}^{0}$ for the system water-potassium chloride $(\mathrm{B})$ - calcium chloride $(\mathrm{C})$ at $25^{\circ} \mathrm{C}$.

Present work, set 7 of table $1, M_{B} \sim 3.44$

Kirgintsev and Luk'yanov, [12], $M_{B}=3.362$

represented by eq (19). As figure 7 shows, the agreement between the two sets of data is good.

These authors had 54 results for mixed solutions but nine of these were for solutions whose total ionic strength exceeded 5 . If we exclude these on the ground that our eq (23) is not applicable at such high total ionic strengths, it is found that the remaining 45 results can be well represented by eq (26), with the $\beta_{0}$ values given earlier. The standard deviation is 0.0021 in $\varphi$, which is also the standard deviation of our own results. 
The excess free energy of mixing is the increase in free energy corresponding to the process

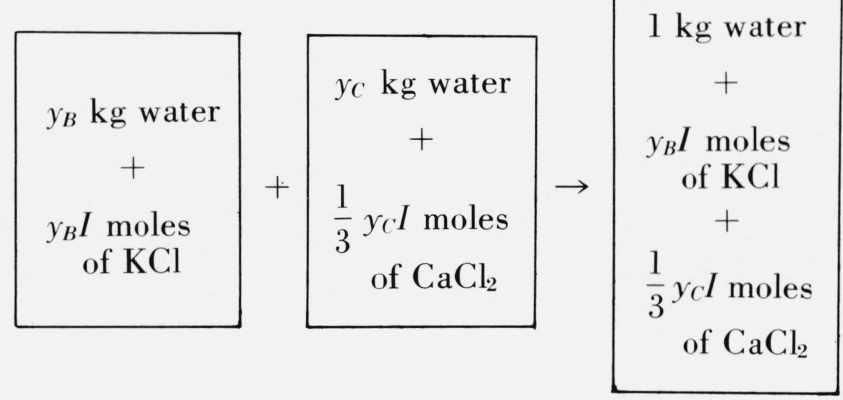

when solutions of potassium chloride and calcium chloride are mixed without change in total ionic strength. As was shown earlier [13], this can be written

$$
\begin{aligned}
G^{E} / R T=y_{B} y_{C} I & \frac{1}{2}\left(Q_{B}+Q_{C}\right)+y_{B} R_{B} \\
& \left.+y_{C} R_{C}+\frac{2}{3}\left(y_{B}-y_{C}\right)\left(R_{C}-R_{B}\right)\right] .
\end{aligned}
$$

Under the conditions represented by eqs (26), (27), and (28), this reduces to the particularly simple form

$$
G^{E} / R T=y_{B} y_{C} I \beta_{0} .
$$

The following values of the excess free energy of mixing in the potassium chloride-calcium chloride system have been calculated by means of eq (30) (in cal per $\mathrm{kg}$ of water, $1 \mathrm{cal}=4.1840 \mathrm{~J}$.) for the case when $y_{B}=y_{C}=0.5$ :

\begin{tabular}{crrrrr}
\multicolumn{1}{c}{$I=$} & \multicolumn{1}{c}{2} & \multicolumn{1}{c}{3} & \multicolumn{1}{c}{4} & \multicolumn{1}{c}{5} \\
$\mathrm{KCl}^{-\mathrm{CaCl}_{2}}$ & 3.4 & 3.7 & 1.0 & -4.8 & -13.7 \\
$\mathrm{KCl}-\mathrm{BaCl}_{2}$ & -2.9 & -12.3 & -35.3 & -73.4 & $\ldots \ldots .$. \\
${\mathrm{NaCl}-\mathrm{CaCl}_{2}}_{2}$ & 3.5 & 15.3 & 30.9 & 50.8 & 75.9 \\
$\mathrm{NaCl}-B a C l_{2}$ & -1.4 & -7.1 & -16.7 & -34.9 & -64.4
\end{tabular}

Some values for other systems of like charge type are included. In general, the excess free energy of mixing is small for the potassium chloride-calcium chloride system; this contrasts with the comparatively large changes in the activity coefficients as shown in table 3 .
The reason for this is to be found in the magnitudes of the $Q_{B}$ and $Q_{C}$ terms in eqs (16), (17), and (29). Assuming, as is the case with this system, that the contribution of the $R$ terms is small compared with that of the $Q$ terms, we see that it is the individual $Q_{B}$ and $Q_{C}$ terms which determine the change in the activity coefficients on mixing, whereas it is the sum $\left(Q_{B}+Q_{C}\right)$ which determines the excess free energy of mixing. It is quite possible that $Q_{B}$ and $Q_{C}$ could be very large, with large changes in the activity coefficients on mixing, yet if $Q_{B}=-Q_{C}$ there would be no change in free energy on mixing.

We thank Dr. J. S. Johnson and Dr. R. M. Rush for many useful discussions and Dr. Rush for the least square calculations on which this paper is based. Our thanks are expressed to the University of Newcastle upon Tyne for the grant of study leave to one of us (A.K.C.) and to the Office of Saline Water for a grant in partial support of this work.

\section{References}

[1] R. A. Robinson and V. E. Bower, J. Res. NBS 69A (Phys. and Chem.), No. 1, 19 (1965).

[2] R. A. Robinson and V. E. Bower, J. Res. NBS 69A (Phys. and Chem.), No. 5, 439 (1965).

[3] R. A. Robinson and V. E. Bower, J. Res. NBS 70A (Phys. and Chem.), No. 4, 313 (1966).

[4] H. A. C. McKay and J. K. Perring, Trans. Faraday Soc. 49, 163 (1953).

[5] G. Scatchard, J. Am. Chem. Soc. 83, 2636 (1961).

[6] H. S. Harned and B. B. Owen, The Physical Chemistry of Electrolytic Solutions, ch. 14, 3d ed. (Reinhold Publ. Corp., New York, N.Y., 1958).

[7] R. A. Robinson and R. H. Stokes, Electrolyte Solutions, ch. 15 , revised ed. (Butterworths Scientific Publications, London, 1965).

[8] R. A. Robinson, J. Phys. Chem. 65, 662 (1961).

[9] R. M. Rush and R. A. Robinson, J. Tenn. Acad. Sci., 43, 22 (1968).

[10] Reference [7], p. 476.

[11] Reference [7], p. 478.

[12] A. N. Kirgintsev and A. V. Luk'yanov, Russ. J. Phys. Chem. 40, 686 (1966).

[13] See appendix to reference [2].

(Paper 72A3-497) 